\title{
Paraplegia
}

\section{Control of Detrusor Hyperreflexia by the Intravesical Instillation of Oxybutynine Hydrochloride}

\author{
H. Madersbacher, $\mathrm{MD},{ }^{1} \mathrm{G}$. Jilg, $\mathbf{M D}^{2}$ \\ ${ }^{1}$ Department of Urology, University Hospital, Innsbruck ${ }^{2}$ Rehabilitation Center, Bad \\ Häring, Tirol, Austria.
}

\begin{abstract}
Summary
This report deals with the control of detrusor hyperreflexia by the intravesical instillation of oxybutynin hydrochloride $(\mathrm{OH})$ in 10 male and 3 female patients with complete suprasacral spinal cord lesions having clean intermittent catheterisation (CIC) because of unbalanced voiding. The indication for intravesical $\mathrm{OH}$ application was persisting urinary incontinence despite CIC in 11 patients and in 2 patients detrusor hypercontractility. One $5 \mathrm{mg}$ tablet of $\mathrm{OH}$ was dissolved in distilled water and the solution was instilled into the bladder through the catheter, which has been used for urodynamics and which was then removed. Six hours later cystometry was repeated and the clinical effects were studied especially with regard to continence/incontinence and side-effects. The differences in the cystometric bladder capacity and maximum detrusor pressure before and after instillation of $\mathrm{OH}$ are statistically highly significant. Clinically, from those 10 patients who were incontinent between CIC before, 9 remained dry during the 6-hour period. None of the patients reported any side-effect after intravesical application of OH. However, with subsequent oral medication 8 out of 12 patients complained of typical anticholinergic side-effects. These results indicate that treatment with topical $O H$ is an effective alternative to treating detrusor hyperreflexia, especially in patients already on CIC because of unbalanced voiding, but with persisting urinary incontinence due to detrusor hyperreflexia. $\mathrm{OH}$ is well absorbed from the bladder, however absorption seems to be protracted compared to oral intake.
\end{abstract}

Key words: Intravesical oxybutynin; Detrusor hyperreflexia; Neurogenic urinary incontinence

Hyperreflexia of the detrusor combined with hyperreflexia of the striated sphincter is typical for the so-called reflex bladder and the uninhibited neuropathic bladder. The urodynamic characteristics are uncontrolled, sometimes hypercontractile and unsustained detrusor contractions together with a functional outflow obstruction mostly at the level of the striated sphincter (detrusor-sphincter dyssynergia). Clinically these findings may result in an unbalanced micturition with a high risk for upper urinary tract damage by reflux and dilatation and in urinary incontinence 


\title{
Paraplegia
}

\section{Control of Detrusor Hyperreflexia by the Intravesical Instillation of Oxybutynine Hydrochloride}

\author{
H. Madersbacher, $\mathrm{MD},{ }^{1} \mathrm{G}$. Jilg, $\mathbf{M D}^{2}$ \\ ${ }^{1}$ Department of Urology, University Hospital, Innsbruck ${ }^{2}$ Rehabilitation Center, Bad \\ Häring, Tirol, Austria.
}

\begin{abstract}
Summary
This report deals with the control of detrusor hyperreflexia by the intravesical instillation of oxybutynin hydrochloride $(\mathrm{OH})$ in 10 male and 3 female patients with complete suprasacral spinal cord lesions having clean intermittent catheterisation (CIC) because of unbalanced voiding. The indication for intravesical $\mathrm{OH}$ application was persisting urinary incontinence despite CIC in 11 patients and in 2 patients detrusor hypercontractility. One $5 \mathrm{mg}$ tablet of $\mathrm{OH}$ was dissolved in distilled water and the solution was instilled into the bladder through the catheter, which has been used for urodynamics and which was then removed. Six hours later cystometry was repeated and the clinical effects were studied especially with regard to continence/incontinence and side-effects. The differences in the cystometric bladder capacity and maximum detrusor pressure before and after instillation of $\mathrm{OH}$ are statistically highly significant. Clinically, from those 10 patients who were incontinent between CIC before, 9 remained dry during the 6-hour period. None of the patients reported any side-effect after intravesical application of OH. However, with subsequent oral medication 8 out of 12 patients complained of typical anticholinergic side-effects. These results indicate that treatment with topical $O H$ is an effective alternative to treating detrusor hyperreflexia, especially in patients already on CIC because of unbalanced voiding, but with persisting urinary incontinence due to detrusor hyperreflexia. $\mathrm{OH}$ is well absorbed from the bladder, however absorption seems to be protracted compared to oral intake.
\end{abstract}

Key words: Intravesical oxybutynin; Detrusor hyperreflexia; Neurogenic urinary incontinence

Hyperreflexia of the detrusor combined with hyperreflexia of the striated sphincter is typical for the so-called reflex bladder and the uninhibited neuropathic bladder. The urodynamic characteristics are uncontrolled, sometimes hypercontractile and unsustained detrusor contractions together with a functional outflow obstruction mostly at the level of the striated sphincter (detrusor-sphincter dyssynergia). Clinically these findings may result in an unbalanced micturition with a high risk for upper urinary tract damage by reflux and dilatation and in urinary incontinence 
due to the lack of voluntary detrusor control. The aim of therapy in these patients is (1) to protect the upper urinary tract by adequate bladder emptying, and (2) to prevent urinary incontinence if possible.

Adequate bladder emptying can be achieved by clean intermittent (self-) catheterisation (CIC), but urinary incontinence in between may persist in the presence of detrusor hyperreflexia. The management of hyperreflexic voiding dysfunction with secondary detrusor incontinence includes the treatment with anticholinergic and spasmolytic drugs, such as oxybutynine hydrochloride $(\mathrm{OH})$, which like others is given orally. Unfortunately many patients, especially the elderly, suffer from unacceptable anticholinergic side-effects when taking these drugs. Dryness of the mouth, disturbance of accommodation and increased constipation, which is clinically relevant especially in persons who already suffer simultaneously from a neurogenic bowel and rectal dysfunction. If one accepts the concept of pharmacological relaxation of the detrusor together with CIC as the method of choice to achieve bladder emptying and to prevent urinary incontinence in between the catheterisations, the question is, how to decrease or to prevent anticholinergic side-effects with these drugs. One way would be the instillation of the drug into the bladder itself. Such an intravesical application seems to be logical especially for patients already on CIC, as the drug could easily be instilled regularly at the end of catheterisation without additional instrumentation.

The aim of the study was to find out (1) whether $\mathrm{OH}$ is effective when given intravesically and (2) if general side-effects do or do not occur.

\section{Material and methods}

All 13 patients, 10 males and 3 females, age range between 19 and 60 years, with a mean age of 30 years had a complete suprasacral spinal cord lesion. Due to an unbalanced voiding all 13 were on CIC. The indication for therapy with $\mathrm{OH}$ was in 11 patients persisting urinary incontinence despite CIC, and in 2 patients hypercontractility of the detrusor with a maximum detrusor pressure above $100 \mathrm{~cm}$ $\mathrm{H}_{2} \mathrm{O}$. The cystometric bladder capacity ranged between 50 and $400 \mathrm{cc}$, the values of the maximal detrusor pressure between 30 and $180 \mathrm{cc}$ (Table I).

Water cystometry with a filling rate of $50 \mathrm{cc} /$ minute with physiological sodium chloride at room temperature as the filling medium was performed to evaluate the activity and the contractility of the detrusor in all patients before the trial was started.

Commercially available $5 \mathrm{mg} \mathrm{OH}$ tablets were crushed and the powder dissolved in $30 \mathrm{cc}$ distilled water. The solution was instilled into the bladder through the catheter which has been used for urodynamics, then the catheter was removed. Six hours later cystometry was repeated. A statistical analysis in regard to the change of cystometric bladder capacity and of the maximum detrusor pressure was done with a standard one way analysis of variance. Moreover the patients were asked about the clinical effects especially with regards to continence/incontinence and regarding general side-effects.

To evaluate whether or to what degree $\mathrm{OH}$ is absorbed from the bladder, a study on the serum levels of $\mathrm{OH} 3,6$ and 9 hours after its intravesical application was undertaken and compared to those after oral intake (Herchulez et al., 1986). 
Table I Intravesical application of oxybutynine: urodynamic results, cystometric bladder capacity and detrusor pressure $\left(\mathrm{cm} \mathrm{H}_{2} \mathrm{O}\right)$ before and 6 hours after intravesical application of $5 \mathrm{mg}$ oxybutynine solution

\begin{tabular}{|c|c|c|c|c|}
\hline \multirow[b]{2}{*}{$\begin{array}{l}\text { Patient } \\
\text { age/sex }\end{array}$} & \multicolumn{2}{|c|}{ Cystometric bladder capacity (CC) } & \multicolumn{2}{|c|}{$\begin{array}{l}\text { Maximal detrusor pressure } \\
\qquad\left(\mathrm{CM} \mathrm{H}_{2} \mathrm{O}\right)\end{array}$} \\
\hline & $\begin{array}{c}\text { Before } \\
\text { instillation }\end{array}$ & $\begin{array}{c}6 \text { hours after } \\
\text { instillation of } \\
5 \mathrm{mg} \mathrm{OH}\end{array}$ & $\begin{array}{c}\text { Before } \\
\text { instillation }\end{array}$ & $\begin{array}{l}6 \text { hours after } \\
\text { instillation }\end{array}$ \\
\hline $41 \mathrm{~F}$ & 120 & 400 & 30 & 20 \\
\hline $46 \mathrm{M}$ & 350 & 500 & 40 & 0 \\
\hline $26 \mathrm{M}$ & 250 & 400 & 65 & 15 \\
\hline $41 M$ & 150 & 100 & 150 & 140 \\
\hline $20 \mathrm{M}$ & 300 & 450 & 55 & 0 \\
\hline $60 \mathrm{M}$ & 50 & 80 & 40 & 30 \\
\hline $41 \mathrm{~F}$ & 120 & 400 & 90 & 60 \\
\hline $25 \mathrm{~F}$ & 200 & 400 & 85 & 30 \\
\hline $19 M$ & 200 & 400 & 60 & 30 \\
\hline $19 M$ & 200 & 400 & 55 & 45 \\
\hline $23 M$ & 400 & 450 & 60 & 60 \\
\hline $20 M$ & 170 & 230 & 180 & 55 \\
\hline $19 M$ & 100 & 450 & 45 & 30 \\
\hline
\end{tabular}

A prerequisite for the study was sterile urine and a leucocyte count below $50 / \mathrm{cmm}$.

\section{Results}

\section{Cystometric findings}

Ten of 13 patients showed a decrease of the maximum detrusor pressure 6 hours after instillation of $\mathrm{OH}$, which ranged between 125 and $10 \mathrm{~cm} \mathrm{H}_{2} \mathrm{O}$ with a mean decrease of $36 \mathrm{~cm} \mathrm{H}_{2} \mathrm{O}$. In these 10 patients likewise the cystometric bladder capacity increased considerably between 30 and $280 \mathrm{cc}$ (Fig. 1, Fig. 2), with a mean value of $141.6 \mathrm{cc}$. The differences in the cystometric bladder capacity and in the maximum detrusor pressure before and after intravesical instillation of $\mathrm{OH}$ are statistically highly significant with a PR $>\mathrm{T}$ of 0.0005 for bladder capacity and 0.0043 for maximum detrusor pressure. Of those 2 patients in whom a increased maximum detrusor pressure was the indication for this therapy, one responded, the other not. The results are summarised in the Table.

\section{Clinical symptomatology}

From those 11 patients, who were incontinent in the intervals between CIC before, 10 remained dry during the 6-hour period following intravesical instillation of $\mathrm{OH}$. This clinical effect correlated well with the urodynamic findings.

None of the patients reported any side-effects after intravesical application of $\mathrm{OH}$. After the study 12 of the 13 patients received $\mathrm{OH}$ orally. With subsequent oral medication 9 of the 12 patients complained of typical anticholinergic sideeffects.

Those who responded to intravesical application also responded to oral therapy, 


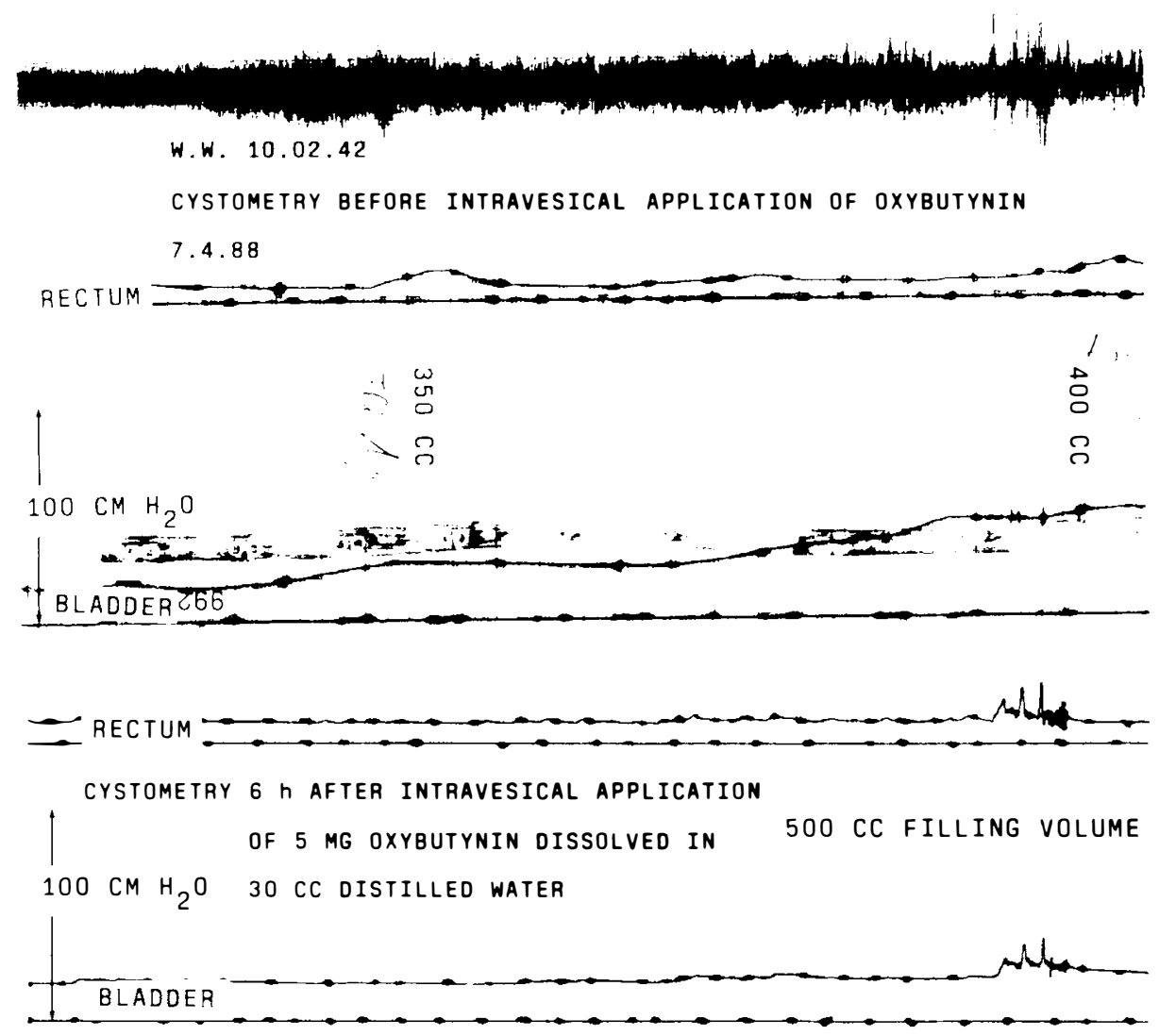

Figure 1 Urodynamic investigation of a 46-year-old male patient (A) before and (B) 6 hours after intravesical application of $5 \mathrm{mg}$ oxybutynine dissolved in $30 \mathrm{cc}$ distilled water: the detrusor became acontractile, the bladder capacity increased from 350 to $500 \mathrm{cc}$; the upper curve in each of the 2 urodynamic studies represent the intrarectal, the lower curve the intravesical pressure

whereas the 2 local non-responders were oral non-responders as well.

\section{Plasma concentrations of $\mathrm{OH}$ after intravesical application}

The plasma concentrations of $\mathrm{OH} 3,6$ and 9 hours after intravesical application of $\mathrm{OH}$ were evaluated in 5 patients with normal bladder function. The results indicate that $\mathrm{OH}$ is at least partially absorbed from the bladder, however, the absorption seems to be protracted compared to oral intake (Fig. 3).

\section{Discussion}

The aim in the therapy of detrusor hyperreflexia is to protect the upper urinary tract by adequate bladder emptying and to prevent urinary incontinence due to involuntary bladder contractions. These goals may be achieved by several therapeutic strategies. Sphincterotomy, which is still popular to achieve balanced voiding in males with an unbalanced reflex bladder, certainly protects the upper 

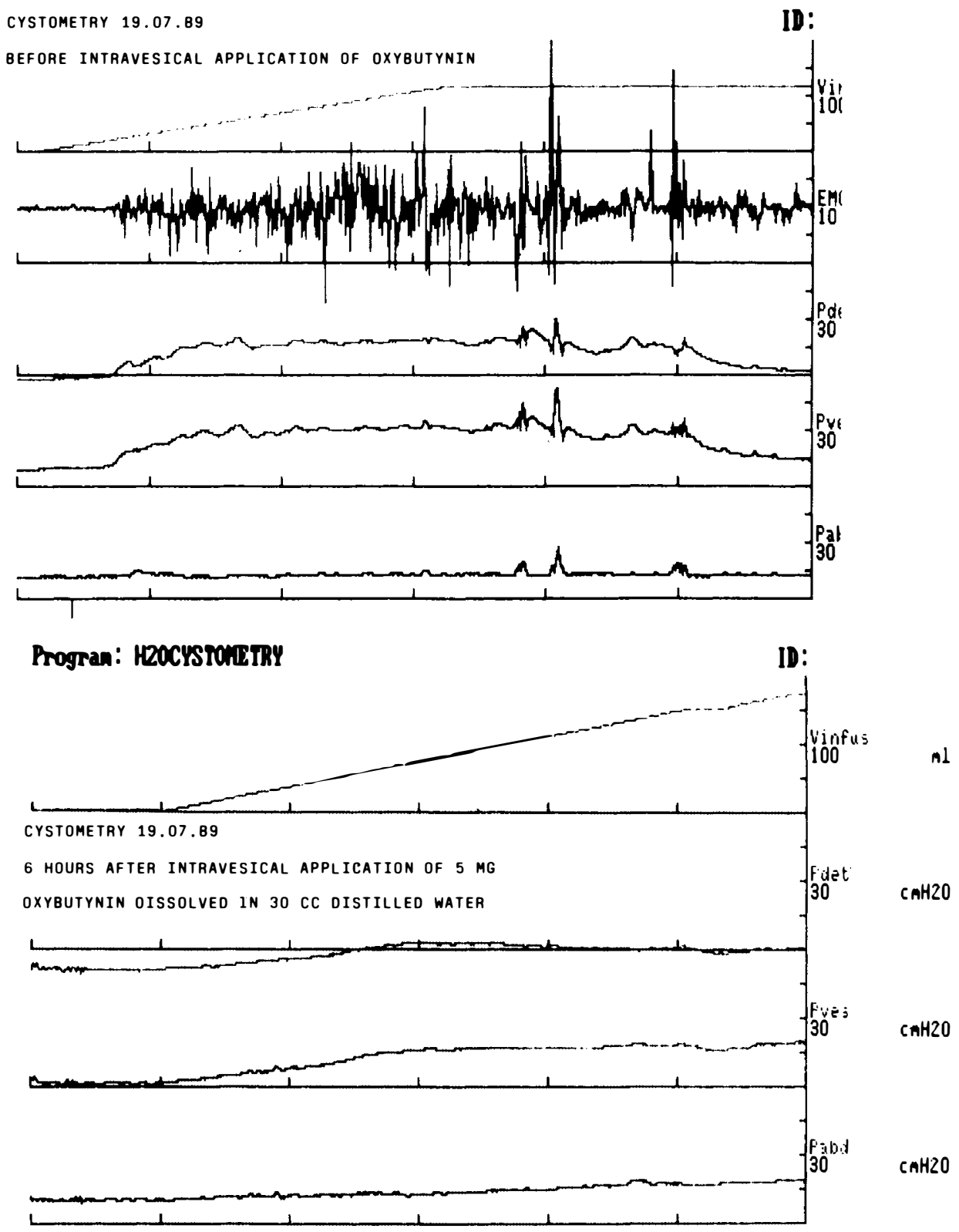

Figure 2 Urodynamic investigation of a 19-year-old male patient (A) before and (B) after intravesical application of $5 \mathrm{mg}$ oxybutynine. The maximum detrusor pressure decreased from 60 to $30 \mathrm{~cm} \mathrm{H}_{2} \mathrm{O}$, the cystometric bladder capacity increased from 200 to $400 \mathrm{cc}$, the patient remained dry in between

urinary tract, but it does not solve the problem of incontinence. With a posterior sacral rhizotomy the bladder becomes hyporeflexic or areflexic, bladder emptying has to be achieved either with CIC or by implanting an anterior sacral root stimulator (Brindley, et al., 1982). Detrusor hyperreflexia can also be managed by bladder augmentation using an ileum patch (clam-ileocystoplasty) which acts as 


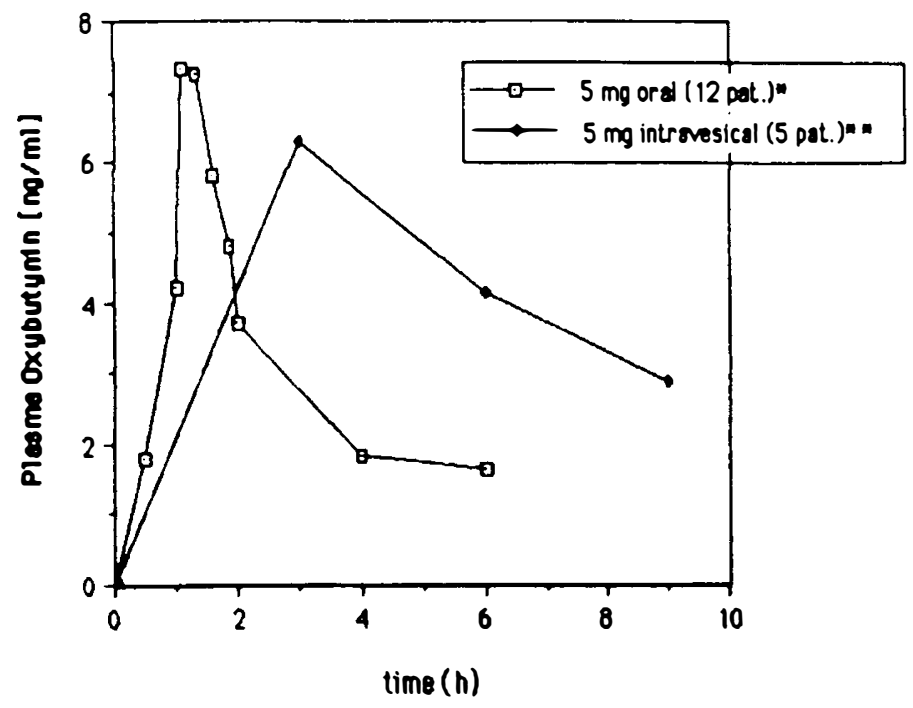

Figure 3 Plasma oxybutynine (ng/ml) after intravesical application of $5 \mathrm{mg}$ oxybutynine solution, mean values of 5 patients (own results) compared to plasma oxybutynine after oral intake, mean values of 12 volunteers. ( ${ }^{\star}$ Herchulez et al., $1986{ }^{\star \star}$ Madersbacher, unpublished results).

an energy destroyer for the unvoluntary detrusor contractions. However, posterior sacral root rhizotomy as well as bladder augmentation are major operations, therefore pharmacological relaxation of the detrusor is still the method of choice.

$\mathrm{OH}$ is a tertiary amine with a comparatively weak anticholinergic, but a potent local anaesthetic and effective spasmolytic effect on a variety of smooth muscle tissues. In animal studies the spasmolytic effect was 2 times greater than that of probantheline bromide and 10 times greater than that of atropine (Fredericks $e t$ al., 1975). $\mathrm{OH}$ is absorbed readily from the gastrointestinal tract. Since Diokno and Lapides (1972) reported successful results with $\mathrm{OH}$ to treat detrusor hyperreflexia numerous subsequent trials have demonstrated the efficacy of this drug in patients with detrusor hyperreflexia (Thompson and Lauretz, 1976; Mulcahy et al., 1977; Thüroff et al., 1988).

Side-effects due to the anticholinergic properties of the drug, are more pronounced in elderly people and comprise primarily dry mouth and, less often, nausea, gastric disorders, drowsiness and blurred vision. According to Thüroff $e t$ al. (1988) side-effects were reported in $63 \%$ of patients half being classified severe, but only $3 \%$ of the drop-outs in this trial were due to side-effects.

In order to reduce the side-effects and to concurrently optimise the beneficial effects of $\mathrm{OH}$, we tested the effect of intravesical administration of this agent. Patients who were already on CIC without achieving urinary incontinence, were regarded as especially suitable for intravesical application of the drug, as the drug could be instilled into the bladder without additional instrumentation, as part of routine catheterisation. In a previous study Mohler (1987) had already evaluated the application of intravesical $\mathrm{OH}$ in canine intestinal bladders, he found, that $\mathrm{OH}$ achieved better bladder relaxation when administered intravesically.

Our pilot study demonstrates that intravesical instillation of $\mathrm{OH}$ is effective in patients with urinary incontinence due to detrusor hyperreflexia without causing 
the usual general side-effects of the drug. Our results confirm those, recently published by Brendler et al. (1989), who treated 13 patients with detrusor hyperactivity, secondary incontinence and with frequent side-effects on oral anticholinergic agents by applying $\mathrm{OH}$ topically twice daily via an introduced catheter. Ten of them experienced subjective improvements in urinary symptoms and all became continent following treatment, 6 of them continued the procedure.

In regard to the mode of action of $\mathrm{OH}$ applied intravesically our preliminary results in a small number of persons let us assume that the effect of topical $\mathrm{OH}$ is at least partially a systemic one. The absorption rate seems to be lower compared to oral intake, but more data are necessary are to clarify the situation. We are currently investigating the serum levels of $\mathrm{OH}$ also 1 and 2 hours after intravesical application. It may well be, that this protracted absorption is the reason for the lack of side effects with intravesical application. However, further investigations are necessary to explain these findings.

Interesting enough, in our series those who responded to intravesical application also responded to oral therapy with $\mathrm{OH}$, whereas local non-responders were oral non-responders as well. Therefore the effect of $\mathrm{OH}$, applied intravesically during a urodynamic investigation, on detrusor hyperreflexia may also be of prognostic value for the efficiency of subsequent possible oral intake of $\mathrm{OH}$. However, this suggestion also needs further confirmation in more patients.

From our experience, intravesical instillation of $\mathrm{OH}$ is especially suitable for patients, who are already on CIC but are still incontinent in between due to persisting detrusor hyperreflexia. One of our patients used intravesical application of $\mathrm{OH}$ during 3 months and maintained the initial favourable effect of this therapy. However, in regard to clinical practicability and for a wider use of intravesical application of $\mathrm{OH}$, a solution of $5 \mathrm{mg} \mathrm{OH}$, in a disposable package with a suitable attachment to the catheter should be available.

\section{References}

BRENDLER BCH, RAdebaugh, LC, MoHLER JL 1989 Topical oxybutynin chloride for relaxation of dysfunctional bladders. Fournal of Urology 141:1350-1352.

BRINDLEY GS, PolKeY CE, RuShTON DN 1982 Sacral anterior root stimulators for bladder control in paraplegia. Paraplegia 20:365-381.

DiOKNO AC, LAPIDES J 1972 Oxybutynin: a new drug with analgesic and anticholinergic properties. Fournal of Urology 108:307-309.

FREDERICKS CM, ANDERSON GF, KREULEN, DL 1975 A study of the anticholinergic and antispasmodic activity of oxybutynin (Ditropan) on rabbit detrusor. Investigative Urology 12: 317-319.

MulCAHY JJ, James HE, MCROBERTS JW 1977 Oxybutynin chloride combined with intermittent clean catheterization in the treatment of myelomeningocele patients. Fournal of Urology 118:9596.

MOHLER JL 1987 Relaxation of intestinal bladder substitutes and neurogenic bladder by intravesical delivered pharmaceutics. Fournal of Urology 137: No.4, Part 2, p 108A.

MOHLER JL: Cit. by Brendler et al. 1989 Relaxation of intestinal bladders by intravesical oxybutynin chloride. Fournal of Urology 141:1350-1352.

THOMPSON IM, LAURETZ R 1976 Oxybutynin in bladder spasm, neurogenic bladder and enuresis. Urology 8:452-454.

ThÜroff JW, BUNKE B, Ebner A et al. 1990 Randomized double blind multicenter trial on treatment of frequency, urgency and incontinence related to detrusor hyperactivity: Oxybutynin versus Propantheline versus placebo. Fournal of Urology (in press). 